\title{
Erratum to: The Relationship between Formaldehyde Exposure Levels and Environmental Diseases within Residential Environments
}

\author{
Je-Gyu Hwang ${ }^{1}$, Im-Soon Kim² \& Eun-Young Lee ${ }^{3}$ \\ ${ }^{1}$ EHS Technical Research Center \\ ${ }^{2}$ Graduate School of Environmental Studies, Kwangwoon University \\ ${ }^{3}$ Department of Environmental Engineering, University of Suwon \\ Correspondence and requests for materials should be addressed \\ to E.-Y. Lee (ley@suwon.ac.kr) \\ DOI 10.1007/s13530-015-0230-4 \\ (C) The Korean Society of Environmental Risk Assessment and \\ Health Science and Springer 2015
}

In the 2015 issue of Toxicology and Environmental Health Sciences (ToxEHS), an error occurred in the research article. Je-Gyu Hwang, Im-Soon Kim, EunYoung Lee (2015) The Relationship between Formaldehyde Exposure Levels and Environmental Diseases within Residential Environments. Toxicol. Environ. Health Sci. 7, 124-128.
-In the authors's affiliation

Original:

Im-Sun Kim

Graduate School of Environmental Studies, Kwangwoon University

This should be replaced by:

Im-Soon Kim

Graduate School of Environmental Studies, Kwangwoon University 